\title{
Temperature and entropy profiles of nearby cooling flow clusters observed with XMM-Newton
}

\author{
R. Piffaretti ${ }^{1,2}$, Ph. Jetzer ${ }^{1}$, J. S. Kaastra ${ }^{3}$, and T. Tamura ${ }^{3,4}$ \\ ${ }^{1}$ Institute of Theoretical Physics, University of Zürich, Winterthurerstrasse, 190, 8057 Zürich, Switzerland \\ e-mail: piff@physik.unizh.ch \\ 2 Laboratory for Astrophysics, Paul Scherrer Institute, 5232 Villigen PSI, Switzerland \\ 3 SRON National Institute for Space Research, Sorbonnelaan 2, 3584 CA Utrecht, The Netherlands \\ ${ }^{4}$ Institute of Space and Astronautical Science, 3-1-1 Yoshinodai, Sagamihara, Kanagawa 229-8510, Japan
}

Received 24 August 2004 / Accepted 1 December 2004

\begin{abstract}
We investigate temperature and entropy profiles of 13 nearby cooling flow clusters observed with the EPIC cameras of XMM-Newton. When normalized and scaled by the virial radius the temperature profiles turn out to be remarkably similar. At large radii the temperature profiles show a clear decline starting from a break radius at $\sim 0.1 r_{\text {vir }}$. The temperature decreases by $\sim 30 \%$ between $0.1 r_{\text {vir }}$ and $0.5 r_{\text {vir }}$. As expected for systems where non-gravitational processes are of great importance, the scale length characterizing the central temperature drop is not found to be proportional to the virial radius of the system. The entropy of the plasma increases monotonically moving outwards almost proportional to the radius and the central entropy level is tightly correlated with the core radius of the X-ray emission. The dispersion in the entropy profiles is smaller if the empirical relation $S \propto T^{0.65}$ is used instead of the standard self-similar relation $S \propto T$ and, as expected for cooling flow clusters, no entropy cores are observed.
\end{abstract}

Key words. galaxies: clusters: general - cooling flows - X-rays: galaxies: clusters

\section{Introduction}

Temperature profiles of the X-ray-emitting intracluster medium (ICM) have several important implications. Derived from $\mathrm{X}$-ray data together with the gas density distribution, they are critical in deriving the total gravitational mass of galaxy clusters, which combined with the estimated gas mass and primordial nucleosynthesis calculations, provides constraints on the cosmological density parameter (e.g., White et al. 1993). Temperature profiles provide useful knowledge to complementary observations such as the Sunyaev-Zel'dovich effect and affect the determination of cosmological parameters like the Hubble constant (e.g., Rephaeli 1995). They also provide valuable information on the thermodynamic history of galaxy clusters, since they are fundamental in determining the ICM entropy distribution, and the latter has been shown to be a very powerful tool to study non-gravitational processes such as radiative cooling, preheating and feedback from supernovae and active galactic nuclei (e.g., Voit 2004).

Various observational studies have found different and conflicting results regarding temperature gradients in the outer regions of galaxy clusters. Markevitch et al. (1998) analyzed projected radial temperature profiles for 30 clusters observed with ASCA, finding that nearly all the clusters in their sample, including cooling flow clusters, show a significant decline at large radii. Irwin et al. (1999) pointed out that the claim by Markevitch et al. (1998) of a declining temperature profile is in contrast with the findings of other authors even for the same object and strengthened the claim of isothermality by investigating ROSAT PSPC color profiles. White (2000) studied a sample of 106 clusters observed with ASCA and found that 90 percent of the temperature profiles are consistent with isothermality. Irwin \& Bregman (2000) derived radial temperature profiles for 11 clusters observed with BeppoSAX and found generally flat or slightly increasing temperature profiles, therefore ruling out a declining temperature profile. On the other hand Finoguenov et al. (2001b) found declining profiles similar to those of Markevitch et al. (1998) from the analysis of ASCA/SIS data of 18 relaxed cool clusters. De Grandi \& Molendi (2002) analyzed the projected temperature profiles for 11 cooling flow and 10 non-cooling flow clusters observed with BeppoSAX. They find a rapid decline of the profiles beyond $\sim 0.2 r_{180}$ and that non-cooling flow clusters appear to be well described by an isothermal core extending out to $\sim 0.2 r_{180}$, while the well known signature of cooling flow clusters is the temperature decline towards the center. Despite the agreement between Markevitch et al. (1998), Finoguenov et al. (2001b) and De Grandi \& Molendi (2002), the behavior of cluster temperature profiles beyond $\sim 0.2 r_{180}$ remains controversial, mainly because even with large samples the number of clusters where the temperature is derived beyond $0.5 r_{180}$ is very small. 
In the early phases of the XMM-Newton mission, Arnaud et al. (2001) found that the temperature profile of A 1795 is essentially isothermal up to $\sim 0.4 r_{200}$. Pratt et al. (2001) find a flat temperature profile up to $\sim 0.5 r_{200}$ for A 2163 and an indication of a mild decline at larger radii. XMM-Newton observation of the distant cluster RX J1120.1+4318 indicate a flat profile up to $\sim 0.5 r_{\text {vir }}$ (Arnaud et al. 2002), and no sharp temperature drop is found in A 1835 up to $\sim 0.7 r_{\text {vir }}$ (Majerowicz et al. 2002). Pratt \& Arnaud (2002) find a declining temperature profile in A 1413. The gradient is modest compared to the results of Markevitch et al. (1998) and De Grandi \& Molendi (2002): the temperature decreases by $\sim 20 \%$ between $0.1 r_{200}$ and $0.5 r_{200}$. The temperature profile of A 1983 derived within $\sim 0.4 r_{200}$ by Pratt \& Arnaud (2003) does not show a decline and for A 1650 Takahashi \& Yamashita (2003) find a temperature decline of $\sim 20 \%$ between $0.1 r_{200}$ and $0.4 r_{200}$. Given these controversial results on temperature gradients at large radii, any further insight is welcome.

In this paper we thus investigate radial temperature profiles of nearby cooling flow clusters using the results from the spatially resolved spectra taken with the EPIC cameras of XMM-Newton presented in Kaastra et al. (2004). We present an accurate description of temperature and entropy profiles at all the observed radii and investigate their scaling properties. We additionally provide information on the "uncorrected" luminosity-temperature relation for cooling flow clusters.

The layout of this paper is as follows. In Sect. 2 we briefly summarize the procedure and results which are essential for our analysis (see Kaastra et al. 2004, for the description of the sample and the extensive presentation of the data analysis) and discuss the selection of temperature bins. In Sect. 3 we compute, for each cluster, gas and total gravitational masses, and the virial radius needed for the scaling of temperature profiles presented in Sect. 4 along with the comparison with previous work and numerical simulations. In Sect. 5 we focus on the scaling of entropy profiles and present the luminosity temperature relation derived from our sample. In Sect. 6 we summarize the results of our analysis and present the conclusions.

Throughout this work we use, depending on the context, a $\operatorname{SCDM}\left(\Omega_{\mathrm{m}}=1.0, \Omega_{\Lambda}=0.0\right)$ cosmology with $H_{0}=$ $50 \mathrm{~km} \mathrm{~s}^{-1} \mathrm{Mpc}^{-1}$ and/or a $\Lambda \mathrm{CDM}\left(\Omega_{\mathrm{m}}=0.3, \Omega_{\Lambda}=0.7\right)$ cosmology with $H_{0}=70 \mathrm{~km} \mathrm{~s}^{-1} \mathrm{Mpc}^{-1}$. These will be denoted SCDM50 and $\Lambda$ CDM70, respectively. The main reason for presenting results for both cosmologies is that in the literature often results for the SCDM50 cosmology are listed, while only recently more people start using the $\Lambda$ CDM70 model. This makes a comparison of our results with other work more easy.

\section{Sample}

The sample consists of 17 clusters with cooling flows listed in Table 1. Kaastra et al. (2004) derived deprojected (3D) and projected (2D) radial temperature profiles for all the clusters in this sample. From the analysis of deprojected spectra Kaastra et al. (2004) give the results of single-temperature and multi-temperature fittings. Even though the single-temperature model does not provide the best fits to the inner cooling parts in several clusters, we will use the results from the single-phase model for the following reason. The multi-temperature fitting indicates that the gas in the cooling flow region is multiphase and at the same time that the emission measure distribution is dominated by the emission from the hottest gas at each radius (see Kaastra et al. 2004, Table 7). Using the results of the multi-temperature fitting and assuming pressure equilibrium between the gas phases we compute filling factors and total gas density for each shell. We then compute the effective temperature of the gas mixture in each shell and find that, for all clusters and all shells, it agrees within less then a few percents with the shell temperature from the single-temperature modelling.

Spectral fitting is done over the full $0.2-10 \mathrm{keV}$ range. The energy range below $0.2 \mathrm{keV}$ is currently too poorly calibrated to be useful for spectral analysis, while above $10 \mathrm{keV}$ most of our spectra lack sufficient flux. In general, temperatures and gas densities are computed for the innermost 8 shells (see Kaastra et al. 2004, Table 3 for the boundaries between the shells), except when the data become too noisy in the outermost shell.

In the derivation of the total gravitational mass from the 3D temperature and density profiles and for the study of temperature gradients at large radii, it is crucial to select bins with a robust temperature estimate. For Virgo and Perseus the innermost shell is excised because of the presence of a strong X-ray emitting AGN. At high energies, the background as measured with XMM-Newton is dominated by a power law contribution caused by soft protons. This component is time variable and we estimate that we can determine it with a maximum uncertainty of $10 \%$ for each observation. As in the outer regions of the cluster the X-ray flux drops rapidly, this soft proton component dominates (see Kaastra et al. 2004, Sect. 2.3 for a detailed presentation on the background subtraction). For this reason we exclude temperature bins in the outermost parts of the cluster as follows. Considering that the background uncertainty is of the order of $10 \%$, a bin with temperature $k T$ is selected if $10 \%$ of the net flux is larger or equal to $10 \%$ of the background in the energy band containing the turnover region of the bremsstrahlung spectrum, i.e. the one closer to the energy interval $k T-2 k T$. Concerning the choice of the energy band in the selection procedure, we notice that particular carefulness is needed in the selection of the outermost bins with low temperatures. We have applied our selection criterion using different energy bands close to the energy interval $k T-2 k T$ and find that if flux at too low energy compared to $k T$ is taken into account, bins that would otherwise be excluded, satisfy the selection criterion. This effect can be attributed to the soft excess, since it is affecting the outermost bins only and it is strong in clusters known to show soft excess (A 2052, Sérsic 159-3, MKW 9 and MKW 3s). A detailed modelling of the soft excess is beyond the scope of this work and we prefer to exclude bins without robust temperature estimates even though their inclusion would allow us to trace temperature profiles at much larger radii. The selection is performed using both pn and MOS1/2 data. We find the selection with pn to be more conservative than the one performed on MOS1/2 data and therefore use the results of the former. In Table 1 we list $R_{\text {out }}$, the value of the radius at the outer end of the last radial bin considered after the selection. 
Table 1. The radius at the outer end of the last radial bin considered, $R_{\text {out }}$ and the best fit parameters for the NFW dark matter density profile for SCDMH50 and $\Lambda$ CDMH70. Virgo, Hydra A and A 399 do not converge in the $r_{\mathrm{s}}-c$ plane. The quoted errors on $r_{\mathrm{s}}$ and $c$ are rms of the 68 percent joint confidence limits.

\begin{tabular}{|c|c|c|c|c|c|c|c|c|}
\hline \multirow[b]{2}{*}{ Cluster } & \multicolumn{4}{|c|}{ "SCDMH50 } & \multicolumn{4}{|c|}{ 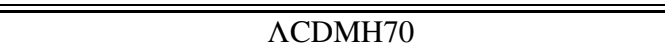 } \\
\hline & $\begin{array}{r}R_{\text {out }} \\
(\mathrm{kpc})\end{array}$ & $\begin{array}{r}r_{\mathrm{s}} \\
(\mathrm{kpc})\end{array}$ & $c$ & $\chi^{2}$ (d.o.f.) & $\begin{array}{r}R_{\text {out }} \\
(\mathrm{kpc})\end{array}$ & $\begin{array}{r}r_{\mathrm{s}} \\
(\mathrm{kpc})\end{array}$ & $c$ & $\chi^{2}$ (d.o.f.) \\
\hline NGC 533 & 267 & $52(4)$ & $12.56(0.56)$ & $3.68(5)$ & 192 & $37(3)$ & $12.53(0.55)$ & $3.78(5)$ \\
\hline Virgo & 56 & - & - & - & 40 & - & - & - \\
\hline A 262 & 316 & $110(22)$ & $9.03(1.03)$ & $8.07(6)$ & 228 & $85(17)$ & $8.62(1.00)$ & $7.73(6)$ \\
\hline A 1837 & 656 & $275(117)$ & $6.07(2.51)$ & $1.07(3)$ & 485 & 211(90) & $5.78(2.66)$ & $1.08(3)$ \\
\hline Sérsic 159-3 & 814 & $209(25)$ & $6.79(0.57)$ & $54.36(5)$ & 598 & $159(18)$ & $6.56(0.54)$ & $53.13(5)$ \\
\hline MKW 9 & 262 & - & - & - & 191 & - & - & - \\
\hline 2 A $0335+096$ & 510 & $228(21)$ & $6.45(0.35)$ & $8.06(5)$ & 371 & $203(20)$ & $5.63(0.33)$ & $13.94(5)$ \\
\hline MKW 3s & 661 & $280(30)$ & $6.19(0.45)$ & $26.60(5)$ & 483 & $213(23)$ & $5.96(0.43)$ & $26.39(5)$ \\
\hline A 2052 & 526 & $311(56)$ & $5.40(0.59)$ & $73.47(5)$ & 383 & $260(48)$ & $4.90(0.55)$ & $87.73(5)$ \\
\hline A 4059 & 675 & $1002(404)$ & $2.76(0.99)$ & $1.92(4)$ & 494 & $744(297)$ & $2.70(1.00)$ & $1.91(4)$ \\
\hline Hydra A (A 780) & 349 & - & - & - & 256 & - & - & - \\
\hline A 496 & 319 & $166(64)$ & $8.11(1.72)$ & $0.46(4)$ & 232 & $129(51)$ & $7.75(1.67)$ & $0.45(4)$ \\
\hline A 3112 & 464 & $462(112)$ & $4.78(2.00)$ & $1.97(3)$ & 344 & $361(166)$ & $4.54(2.04)$ & $1.82(3)$ \\
\hline A 1795 & 900 & $507(57)$ & $4.70(0.32)$ & $14.37(5)$ & 664 & $430(49)$ & $4.21(0.30)$ & $9.04(5)$ \\
\hline A 399 & 983 & - & - & - & 727 & - & - & - \\
\hline Perseus (A 426) & 363 & $629(248)$ & $4.21(0.79)$ & $11.56(4)$ & 261 & 481(189) & $4.09(0.77)$ & $12.30(4)$ \\
\hline A 1835 & 1191 & $799(159)$ & $3.67(0.44)$ & $5.32(3)$ & 950 & $610(150)$ & $3.39(0.41)$ & $5.30(3)$ \\
\hline
\end{tabular}

The inputs of our study are therefore: gas bolometric luminosity, gas density and temperature in each shell within $R_{\text {out }}$.

\section{Gravitational masses}

In order to make a fair comparison between clusters, the various quantities such as gas temperature and entropy must be evaluated at constant fractions of the virial radius, rather than at fixed radii imposed by data limits. We therefore estimate the total mass assuming spherical symmetry and hydrostatic equilibrium and compute $r_{\Delta}$, the radius within which the mean interior density is $\Delta$ times the critical value.

We estimate the total gravitational mass by making direct use of the 3D gas temperature and gas density from the single phase analysis. We invert the equation of hydrostatic equilibrium, i.e.

$M_{\mathrm{tot}}(<r)=-\frac{k T(r)}{\mu m_{\mathrm{p}} G}\left(\frac{\mathrm{d} \ln \rho(r)}{\mathrm{d} \ln r}+\frac{\mathrm{d} \ln T(r)}{\mathrm{d} \ln r}\right) r$,

where $G$ is the gravitational constant, $k$ the Boltzmann constant, $\rho$ the gas density, $T$ its temperature and $\mu m_{\mathrm{p}}$ is the mean particle mass of the gas (we assume $\mu=0.61$ ). Since the mass contribution from galaxies is small we neglect it, and therefore $M_{\mathrm{tot}}(<r)=M_{\mathrm{DM}}(<r)+M_{\mathrm{gas}}(<r)$, i.e. the total gravitational mass within a sphere of radius $r$ is given by gas plus dark matter mass. Using the three-dimensional gas density, we select the dark matter mass model that reproduces better the deprojected temperature profile. As a cosmologically motivated dark matter mass model, we consider the integrated NFW (Navarro et al. 1996) dark matter profile:

$M_{\mathrm{DM}}(<r)=4 \pi r_{\mathrm{s}}^{3} \rho_{\mathrm{c}} \frac{200}{3} \frac{c^{3}\left(\ln \left(1+r / r_{\mathrm{s}}\right)-\frac{r / r_{\mathrm{s}}}{\left(1+r / r_{\mathrm{s}}\right)}\right)}{\ln (1+c)-c /(1+c)}$, where $\rho_{\mathrm{c}}$ is the critical density. The scale radius $r_{\mathrm{s}}$ and the concentration parameter $c$ are the free parameters.

We make use of the NFW dark matter density profile because the choice of an analytical function allows us to extrapolate the dark matter mass profile beyond $R_{\text {out }}$. The best fit parameters that minimize the $\chi^{2}$ of the comparison between the temperatures predicted by Eq. (1) and the observed temperature profile are searched in the intervals $50 \mathrm{kpc}<r_{\mathrm{s}}<1.5 \mathrm{Mpc}$ and $2<c<13$ for both SCDM50 and $\Lambda$ CDM70 cosmologies. The results of the procedure are listed in Table 1 . We have also performed the same fitting procedure by neglecting the gas mass contribution to the total mass and find that in this case the fitting procedure yields substantially larger $\chi^{2}$. We do not obtain any $\chi^{2}$ solution for the Virgo cluster, MKW 9, Hydra A and A 399, which are therefore excluded from the sample. For Virgo we obtain an unphysically small scale radius (and large concentration), because of the small field of view. The failure of the fit for the other clusters can be attributed to the low degree of smoothness of their temperature profiles (no smoothing of the data is used in our procedure).

From the best fit parameters we compute $r_{\Delta}$ using

$\Delta=\frac{3 M_{\mathrm{tot}}\left(<r_{\Delta}\right)}{4 \pi \rho_{c, z} r_{\Delta}^{3}}$

where $\rho_{c, z}=\left(3 H_{z}^{2}\right) /(8 \pi G)$ is the critical density. We estimate $r_{\Delta}$ for various overdensities: $\Delta=$ $2500,2000,1500,1000,500,200$ for both cosmologies, and at $\Delta=\Delta_{\mathrm{c}}$, defined such that $r_{\Delta_{\mathrm{c}}}$ corresponds to the cluster virial radius $r_{\text {vir. }}$. For a SCDM cosmology, $\Delta_{c}=178$, while $\Delta_{\mathrm{c}}=178+82 x-39 x^{2}$ for $\Lambda$ CDM, where $x=\Omega(z)-1$ and $\Omega(z)=\Omega_{\mathrm{m}}(1+z)^{3} /\left(\Omega_{\mathrm{m}}(1+z)^{3}+\Omega_{\Lambda}\right)$ (Bryan \& Norman 1998). 
Table 2. Mean emission-weighted temperature $\left\langle T_{\mathrm{X}}\right\rangle$ and characteristic radii $r_{\Delta}$, dark matter mass $M_{\mathrm{DM}}\left(<r_{\Delta}\right)$ and gas mass $M_{\mathrm{gas}}\left(<r_{\Delta}\right)$ for overdensities $\Delta=2500$ and 500 in the SCDM50 cosmology.

\begin{tabular}{|c|c|c|c|c|c|c|c|}
\hline Cluster & $\begin{array}{r}\left\langle T_{\mathrm{X}}\right\rangle \\
(\mathrm{keV})\end{array}$ & $\begin{array}{r}r_{2500} \\
(\mathrm{kpc})\end{array}$ & $\begin{array}{r}M_{\mathrm{DM}}\left(<r_{2500}\right) \\
\quad\left(10^{13} M_{\odot}\right)\end{array}$ & $\begin{array}{r}M_{\text {gas }}\left(<r_{2500}\right) \\
\left(10^{12} M_{\odot}\right)\end{array}$ & $\begin{array}{r}r_{500} \\
(\mathrm{kpc})\end{array}$ & $\begin{array}{r}M_{\mathrm{DM}}\left(<r_{500}\right) \\
\left(10^{13} M_{\odot}\right)\end{array}$ & $\begin{array}{r}M_{\text {gas }}\left(<r_{500}\right) \\
\left(10^{12} M_{\odot}\right)\end{array}$ \\
\hline NGC 533 & $1.21(0.05)$ & $220(5)$ & $0.81(0.04)$ & $0.56(0.08)$ & $439(12)$ & $1.30(0.09)$ & $1.89(0.27)$ \\
\hline A 262 & $2.17(0.01)$ & $322(21)$ & $2.53(0.34)$ & $2.95(0.42)$ & $663(51)$ & $4.44(0.80)$ & $9.24(1.46)$ \\
\hline A 1837 & $4.00(0.13)$ & $466(51)$ & $9.02(1.77)$ & $9.03(2.16)$ & 1021(208) & $18.99(8.37)$ & $29.08(9.50)$ \\
\hline Sérsic 159-3 & $2.29(0.07)$ & 413(9) & $6.05(0.25)$ & $10.53(0.60)$ & $888(28)$ & $12.02(0.86)$ & $25.17(1.97)$ \\
\hline $2 \mathrm{~A} 0335+096$ & $2.94(0.05)$ & $439(12)$ & $6.79(0.35)$ & $11.69(1.13)$ & $947(32)$ & $13.63(1.03)$ & $26.02(2.79)$ \\
\hline MKW 3s & $3.48(0.14)$ & $503(11)$ & $10.59(0.44)$ & $13.59(0.76)$ & 1095(34) & $21.80(1.51)$ & $35.28(2.27)$ \\
\hline A 2052 & $2.99(0.08)$ & $481(21)$ & $9.00(0.74)$ & $11.33(1.00)$ & $1068(65)$ & $19.63(2.60)$ & $31.64(3.15)$ \\
\hline A 4059 & $3.96(0.05)$ & $640(75)$ & $21.88(3.77)$ & $18.30(3.41)$ & $1637(372)$ & $73.05(30.38)$ & 49.77(12.66) \\
\hline A 496 & $4.20(0.19)$ & $420(48)$ & $5.93(1.37)$ & $9.65(1.96)$ & $880(123)$ & $10.88(3.55)$ & $25.39(5.53)$ \\
\hline A 3112 & $4.33(0.11)$ & $578(104)$ & $17.47(5.18)$ & $20.98(5.15)$ & 1321(390) & $41.64(25.59)$ & $54.49(20.59)$ \\
\hline A 1795 & $5.62(0.04)$ & 631(16) & 22.02(1.02) & $36.29(2.09)$ & $1443(54)$ & $52.58(4.15)$ & $96.49(6.31)$ \\
\hline Perseus (A 426) & $5.30(0.04)$ & $734(89)$ & $30.32(6.41)$ & $26.73(3.77)$ & $1694(272)$ & $74.55(25.13)$ & $53.26(8.18)$ \\
\hline A 1835 & $6.78(0.37)$ & $534(12)$ & $21.78(0.77)$ & $63.18(3.78)$ & $1353(73)$ & $70.93(7.26)$ & $145.52(14.23)$ \\
\hline
\end{tabular}

In the computation of $r_{\Delta}$ we use either 1) $M_{\mathrm{tot}}=M_{\mathrm{DM}}+M_{\mathrm{gas}}$ or, neglecting the gas mass contribution, 2) $M_{\mathrm{tot}}=M_{\mathrm{DM}}$. Assumption 1) naturally leads to values for $r_{\Delta}$ that are larger than those computed assuming 2). For both cosmologies, the relative difference between these values is small: $4-8$ percent for $\Delta=2500$ and $10-15$ percent at $\Delta=500$, depending on the gas mass fraction of the cluster. Even though assumption 1 ) is consistent with our procedure for the dark matter profile estimation, we use the $r_{\Delta}$ values computed considering dark matter only for the following reason. When gas is taken into account, one needs to extrapolate $M_{\text {gas }}(<r)$ for $r>R_{\text {out }}$ when needed. We extrapolate using a least squares fit with an integrated $\beta$-profile with the parameter $\beta$ fixed to values taken from the literature: for Perseus (A 426) from Ettori et al. (1998), for A 3112 from Sanderson et al. (2003) and from Peterson et al. (2003) for the remaining clusters. The mean relative error measured in the observed region is propagated to the extrapolated values. We find that for most of the clusters in our sample extrapolation is needed even for $\Delta=1500$ and that therefore the procedure is very unreliable at large radii. In addition, the extrapolated values for the gas mass are very sensitive to the value of the parameter $\beta$. We therefore prefer, as widely done in the analysis of numerical simulations and in studies similar to our, assumption 2), i.e. we neglect the gas mass contribution when computing $r_{\Delta}$.

The size-temperature relation $r_{\Delta} \propto \sqrt{\left\langle T_{\mathrm{X}}\right\rangle}$ predicted by self-similarity (Mohr \& Evrard 1997) and its normalization provided by catalogues of simulated clusters allows an estimate of $r_{\Delta}$ from the mean emission-weighted temperature $\left\langle T_{\mathrm{X}}\right\rangle$ alone. We employ the widely used predictions given by the simulation of Evrard et al. (1996) and compute the characteristic radii $r_{\Delta}^{\mathrm{Ev}}$. We estimate the mean emission-weighted cluster temperature $\left\langle T_{\mathrm{X}}\right\rangle$ by excluding the cooling flow region (i.e. we excise bins with cooling time less or equal to $15 \mathrm{Gyr}$ ) and fitting the remaining emission-weighted temperature bins with a constant. We list the mean emission-weighted temperatures $\left\langle T_{\mathrm{X}}\right\rangle$ in Table 2. For both SCDM50 and $\Lambda$ CDM70, we find that $r_{\Delta}^{\mathrm{Ev}}$ is systematically larger than the values we compute by directly using the best fit NFW profiles. This is independent on whether our estimate of $r_{\Delta}$ is smaller or larger than $R_{\text {out }}$ and we find that the relative difference can be as high as $60-80$ percent for $\Delta=200$. This result has also been found by Sanderson et al. (2003) with a different method to determine the total gravitational mass.

From the set of $\left(c, r_{\mathrm{s}}\right)$ parameters acceptable at $1 \sigma$ we compute, for each radius $r_{\Delta}$, the maximum and minimum value for the total mass within $r_{\Delta}$. In Tables 2 and 3 we list $r_{\Delta}$, dark matter mass and gas mass within $r_{\Delta}$ for $\Delta=2500$ and 500 for SCDM50 and $\Lambda$ CDM70, respectively. The quoted error related to the mass estimate is the root mean square of the difference between maximum and mean, and the difference between mean and minimum. When extrapolation is needed, the values for the gas mass are computed using the least squares fit described above. We compared our estimates with results from the literature. For clusters with published quantities comparable to ours, we generally find good agreement:

- A 262: Finoguenov et al. (2001a), using temperature profiles derived with ASCA, estimate $r_{500}=860 \pm 170 \mathrm{kpc}$ and $M_{\text {tot }}\left(<r_{500}\right)=9.3 \pm 2.7 \times 10^{13} M_{\odot}$ (in SCDM50). Our estimate of $r_{500}$ (see Table 2) is lower probably due to the fact that contrary to Finoguenov et al. (2001a) we do not include the gas mass contribution in its computation. Consequently also $M_{\text {tot }}\left(<r_{500}\right)$ is lower, but when computing $M_{\text {tot }}(<860 \mathrm{kpc})=6.7 \pm 1.8 \times 10^{13} M_{\odot}$ we find consistency for the total mass estimate.

- 2A 0335+096: as for A 262 the estimate for $r_{500}$ of Finoguenov et al. (2001a) is at variance with our, but agreement is found when the total mass is compared at the same radius: $34.4 \pm 11.3 \times 10^{13} M_{\odot}$ and $26.1 \pm 3.64 \times 10^{13} M_{\odot}$ being Finoguenov et al. (2001a)'s and our estimate, respectively, at $1340 \mathrm{kpc}$. Ettori et al. (2002b) derived total and gas masses from BeppoSAX data at $\Delta=2500$ and 1000 using a method similar to our, with the only difference being that their NFW profile estimates dark matter and gas 
Table 3. Characteristic radii $r_{\Delta}$, dark matter mass $M_{\mathrm{DM}}\left(<r_{\Delta}\right)$ and gas mass $M_{\text {gas }}\left(<r_{\Delta}\right)$ for overdensities $\Delta=2500$ and 500 in the $\Lambda \mathrm{CDM} 70$ cosmology.

\begin{tabular}{lrrrrrr}
\hline \hline Cluster & $r_{2500}$ & $M_{\mathrm{DM}}\left(<r_{2500}\right)$ & $M_{\text {gas }}\left(<r_{2500}\right)$ & $\begin{array}{r}r_{500} \\
\left(10^{13} M_{\odot}\right)\end{array}$ & $\begin{array}{r}M_{\mathrm{DM}}\left(<r_{500}\right) \\
\left(10^{12} M_{\odot}\right)\end{array}$ & $\begin{array}{r}M_{\text {gas }}\left(<r_{500}\right) \\
\left(10^{12} M_{\odot}\right)\end{array}$ \\
\hline NGC 533 & $160(4)$ & $0.59(0.03)$ & $0.25(0.04)$ & $319(9)$ & $0.94(0.06)$ & $0.84(0.12)$ \\
A 262 & $239(16)$ & $1.97(0.27)$ & $1.36(0.20)$ & $495(39)$ & $3.50(0.64)$ & $4.29(0.69)$ \\
A 1837 & $362(42)$ & $7.24(1.51)$ & $4.63(1.14)$ & $793(165)$ & $15.17(6.88)$ & $14.59(4.82)$ \\
Sérsic 159-3 & $320(7)$ & $4.94(0.21)$ & $5.24(0.31)$ & $688(22)$ & $9.77(0.69)$ & $12.28(0.96)$ \\
2A 0335+096 & $344(10)$ & $5.98(0.33)$ & $5.75(0.57)$ & $754(28)$ & $12.60(1.02)$ & $12.73(1.40)$ \\
MKW 3s & $384(8)$ & $8.42(0.36)$ & $6.59(0.37)$ & $835(26)$ & $17.31(1.21)$ & $16.90(1.09)$ \\
A 2052 & $372(17)$ & $7.56(0.65)$ & $5.56(0.50)$ & $833(54)$ & $17.02(2.37)$ & $15.51(1.60)$ \\
A 4059 & $491(62)$ & $17.6(3.25)$ & $8.88(1.73)$ & $1247(304)$ & $57.72(25.74)$ & $23.65(6.31)$ \\
A 496 & $319(37)$ & $4.74(1.12)$ & $4.61(0.95)$ & $668(96)$ & $8.76(2.94)$ & $12.06(2.68)$ \\
A 3112 & $460(86)$ & $14.86(4.63)$ & $10.78(2.74)$ & $1048(317)$ & $35.16(22.24)$ & $27.85(6.40)$ \\
A 1795 & $501(13)$ & $19.05(0.88)$ & $18.71(1.07)$ & $1157(45)$ & $46.87(3.77)$ & $49.18(3.25)$ \\
Perseus (A 426) & $555(68)$ & $24.69(5.22)$ & $12.29(1.72)$ & $1282(207)$ & $61.03(20.59)$ & $24.32(3.72)$ \\
A 1835 & $477(43)$ & $19.93(2.86)$ & $40.46(5.28)$ & $1175(60)$ & $59.61(26.48)$ & $88.05(20.44)$ \\
\hline
\end{tabular}

masses altogether and not dark matter only as in our analysis. Due to this difference and to the larger radial range they access $\left(R_{\text {out }}=917 \mathrm{kpc}\right.$ with respect to $R_{\text {out }}=510 \mathrm{kpc}$ in our work), the best fit scale radius is larger $\left(r_{\mathrm{s}}=626 \mathrm{kpc}\right)$ than our estimate and consequently the concentration parameter smaller. The characteristic radii $r_{\Delta}$ estimated by Ettori et al. (2002b) are also larger than ours, again due to the fact that we neglect the gas mass in the computation. Nevertheless, we find good agreement between the total and gas masses when evaluated at the same physical radius rather than at the same overdensity.

- MKW 3s: we find excellent agreement between the estimates of Finoguenov et al. (2001a), $r_{500}=1210 \pm 140 \mathrm{kpc}$ and $M_{\text {tot }}\left(<r_{500}\right)=25.7 \pm 4.3 \times 10^{13} M_{\odot}$, and ours (see Table 2 for our values of $M_{\mathrm{DM}}$ and $\left.M_{\mathrm{gas}}\right)$.

- A 2052: we find excellent agreement between the estimates of Finoguenov et al. (2001a), $r_{500}=1280 \pm 280 \mathrm{kpc}$ and $M_{\text {tot }}\left(<r_{500}\right)=30.2 \pm 9.6 \times 10^{13} M_{\odot}$ and ours (see Table 2 ).

- A 4059: the values given by Finoguenov et al. (2001a) $\left(r_{500}=1390 \pm 300 \mathrm{kpc}\right.$ and $M_{\mathrm{tot}}\left(<r_{500}\right)=38.7 \pm 12.0 \times$ $10^{13} M_{\odot}$ ) are in agreement with ours (see Table 2 ).

- A 496: as for A 262 and 2A 0335+096 the estimate of $r_{500}$ of Finoguenov et al. (2001a) is at variance with our. Disagreement is still found for the total mass estimated for $r_{500}=1480 \mathrm{kpc}: 47.0 \pm 6.7 \times 10^{13} M_{\odot}$ and $19.9 \pm 9.7 \times$ $10^{13} M_{\odot}$ are Finoguenov et al. (2001a)'s and our estimate, respectively. As for 2A $0335+096$ our estimates can also be compared to Ettori et al. (2002b)'s results. We find discrepancy as in the case of 2A 0335+096: due to the much larger radial range they access $\left(R_{\text {out }}=845 \mathrm{kpc}\right.$ with respect to $R_{\text {out }}=319 \mathrm{kpc}$ in our work), the best fit NFW scale radius is much larger $\left(r_{\mathrm{s}}=738 \mathrm{kpc}\right)$ than our estimate (see Table 1) and consequently the concentration parameter smaller. Furthermore, even when compared at the same radius, our results for both total and gas masses are $\approx 50 \%$ smaller than Ettori et al. (2002b)'s. The disagreement might be due to the fact that the comparison is made at radii much larger than $R_{\text {out }}$, where the mass extrapolation is large.

- A 3112: we find excellent agreement between the estimates of Finoguenov et al. (2001a), $r_{500}=1350 \pm 120 \mathrm{kpc}$ and $M_{\text {tot }}\left(<r_{500}\right)=35.4 \pm 4.5 \times 10^{13} M_{\odot}$ and ours (see Table 2 ).

- A 1795: agreement is found between the estimates of Finoguenov et al. (2001a), $r_{500}=2000 \pm 560 \mathrm{kpc}$ and $M_{\text {tot }}\left(<r_{500}\right)=116.6 \pm 47.2 \times 10^{13} M_{\odot}$ and ours (see Table 2). Better agreement is found when masses are evaluated at the same radius: we estimate $M_{\text {tot }}(<2000 \mathrm{kpc})=$ $82.8 \pm 10.2 \times 10^{13} M_{\odot}$. As for $2 \mathrm{~A} 0335+096$ and A 496 we find a difference between our best fit NFW parameters and those computed by Ettori et al. (2002b) (they can use $R_{\text {out }}=$ $1584 \mathrm{kpc}$ with respect to $R_{\text {out }}=900 \mathrm{kpc}$ in this work). The difference between Ettori et al. (2002b)'s characteristic radii and total and gas masses is nevertheless small and good agreement is found when these quantities are computed at the same radii, i.e. $M_{\mathrm{tot}}(<771 \mathrm{kpc})=40.0 \pm 1.9 \times$ $10^{13} M_{\odot}$ and $M_{\text {gas }}(<771 \mathrm{kpc})=53.7 \pm 0.7 \times 10^{12} M_{\odot}$ from Ettori et al. (2002b) versus $M_{\text {tot }}(<771 \mathrm{kpc})=37.5 \pm 2.5 \times$ $10^{13} M_{\odot}$ and $M_{\mathrm{gas}}(<771 \mathrm{kpc})=47.7 \pm 0.8 \times 10^{12} M_{\odot}$ in this work.

- A 426: even though there is a difference between the NFW parameters estimated by Ettori et al. (2002b) and ours we find excellent agreement between the values of the characteristic radii and both total and gas masses.

- A 1835: Allen et al. (2001) derived total masses from Chandra data at $\Delta=2500$ for both SCDM50 and $\Lambda$ CDM70 and using a method similar to our. For both cosmologies, we find good agreement with their best fit NFW profile parameters and estimated $r_{2500}$. The small differences, in particular the smaller values we obtain for $r_{2500}$, are very likely due to the fact that in their method the gas mass contribution is also included in the computation of $r_{2500}$. When total masses are compared at the same radii, good agreement is found, i.e., 
for SCDM50, $M_{\mathrm{tot}}(<720 \mathrm{kpc})=5.41_{-0.76}^{+1.13} \times 10^{14} M_{\odot}$ and $M_{\text {tot }}(<720 \mathrm{kpc})=4.56 \pm 0.35 \times 10^{14} M_{\odot}$ being Allen et al. (2001)'s value and our estimate, respectively.

- For NGC 533, A 1837 and Sérsic 159-3 a fair comparison with previous work is not possible.

To reiterate, the remainder of our analysis of our final sample of 13 objects relies on the validity of extrapolating profiles fitted to the inner region of the data: for both SCDM50 and $\Lambda \mathrm{CDM} 70$, the number of objects for which the outer radius $R_{\text {out }}$ is larger than (or equal to) $r_{\Delta}$ at the 95 percent level of confidence is: 11 (NGC 533, A 262, A 1837, Sérsic 159-3, 2A 0335+096, MKW 3s, A 2052, A 4059, A 3112, A 1795 and A 1835) for $\Delta=2500,7$ (NGC 533, A 1837, Sérsic 159-3, MKW 3s, A 4059, A 1795 and A 1835) for $\Delta=1500$, only 3 (A 1837, Sérsic 159-3 and A 1835) for $\Delta=1000$ and none for $\Delta=500$.

\section{Scaled temperature profiles}

In the following we investigate the structure of the temperature profiles for our final sample of 13 cooling flow clusters. In Figs. 1 (for SCDM50) and 2 (for $\Lambda$ CDM70), we present the deprojected radial profiles plotted against the radius in units of $r_{\text {vir }}\left(\approx r_{180}\right.$ for SCDM and $\approx r_{101}$ for $\Lambda$ CDM at $\left.z=0\right)$, where the temperature has been normalized by the mean emissionweighted temperature $\left\langle T_{\mathrm{X}}\right\rangle$. From a visual inspection it is evident that a temperature gradient is present at large radii and that when normalized and scaled by the virial radius, temperature profiles are remarkably similar. In addition almost all the individual profiles clearly show a break radius $r_{\mathrm{br}}$, a decrease of temperature from $r_{\mathrm{br}}$ inwards and a decline at radii larger than $r_{\mathrm{br}}$. In the following, we investigate the shape in detail.

\subsection{The break radius}

In the following we compute the break radius of the individual clusters in units of $r_{\text {vir }}$. For each cluster we divide the scaled temperature profiles in two radial intervals: from the innermost bin to the bin $i$ and from the bin $i+1$ to the outermost one. Temperature profiles in each of the two intervals are then fitted using straight lines, power laws and exponential functions. All the nine combinations are used and for each pair of fitting functions, $i$ is varied until the best fit is achieved. Clusters that do not show a clear temperature decrease in the outer region are excluded. These are A 262, A 496 and Perseus. For the remaining clusters the bin $i$ which gives the best fit is independent of the choice of the fitting functions. The break radius $x_{\mathrm{br}}=r_{\mathrm{br}} / r_{\mathrm{vir}}$ is then defined as $\left(x_{\mathrm{i}}+x_{\mathrm{i}+1}\right) / 2$ (where $x_{\mathrm{i}}$ is the distance of the bin $i$ from the center in units of $r_{\text {vir }}$ ) and its uncertainty $\left(x_{\mathrm{i}+1}-x_{\mathrm{i}}\right) / 2$. In Fig. 3 we show the break radius $x_{\mathrm{br}}^{\mathrm{proj}}=$ $r_{\mathrm{br}}^{\mathrm{proj}} / r_{\mathrm{vir}}$ of the projected temperature profiles in SCDM50 as a function of redshift. The mean value of $x_{\mathrm{br}}^{\mathrm{proj}}$ is 0.11 with a standard deviation of 0.01. Using a different method, De Grandi \& Molendi (2002) find 0.20 for the mean value of $x_{\mathrm{br}}^{\mathrm{proj}}$ for a sample of 11 cooling flow and 10 non-cooling flow clusters and a lower value, 0.16 , for the cooling flow clusters only. Taking into account that De Grandi \& Molendi (2002), who also used

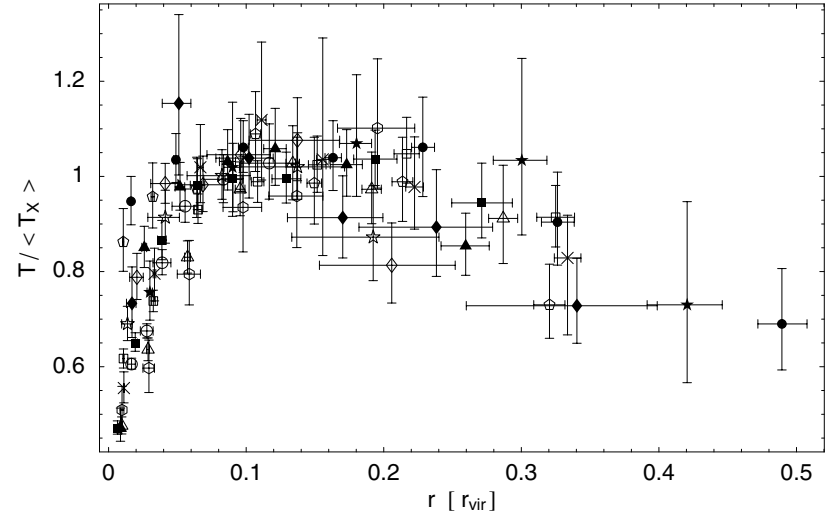

Fig. 1. Scaled temperature profiles (deprojected) in SCDM50 cosmology: the radius is scaled with the virial radius $r_{\mathrm{vir}}=r_{180}$, while the temperature is normalized by the mean emission-weighted temperature $\left\langle T_{\mathrm{X}}\right\rangle$. Clusters are related to symbols as follows: NGC 533 (crosses), A 262 (filled squares), A 1837 (filled diamonds), Sérsic 159-3 (filled circles), 2A 0335+096 (open triangles), MKW 3s (open pentagons), A 2052 (filled triangles), A 4059 (open diamonds), A 496 (open hexagon), A 3112 (open stars), A 1795 (open squares), Perseus (open circles) and A 1835 (filled stars).

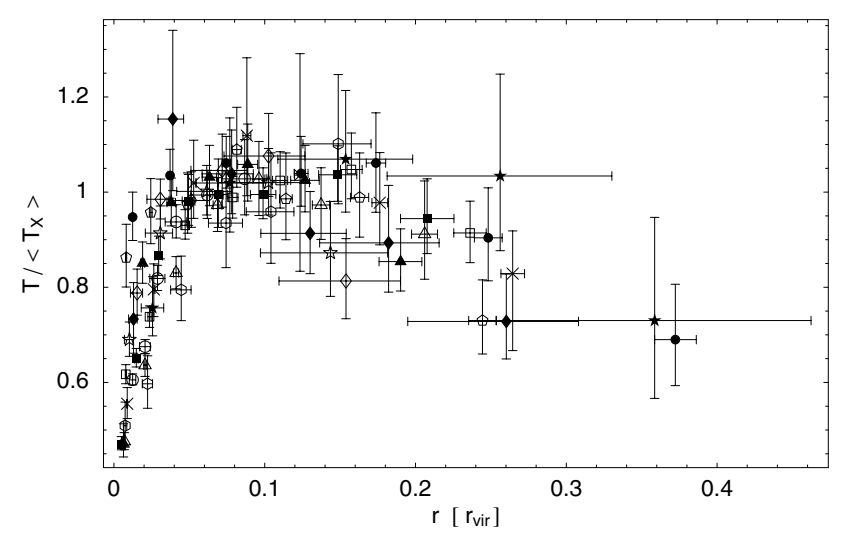

Fig. 2. Same as Fig. 1, but in $\Lambda$ CDM70 cosmology.

projected profiles, computed the break radius for cooling flow clusters by excluding the cooling region and fitting the profiles with a constant temperature for $r<r_{\mathrm{br}}^{\mathrm{proj}}$ and with a line for $r>r_{\mathrm{br}}^{\text {proj }}$, the fact that their estimate is larger than ours is not surprising. In $\Lambda$ CDM70 the mean value of $x_{\mathrm{br}}^{\mathrm{proj}}$ is reduced to 0.08 with a standard deviation of 0.01 . In agreement with De Grandi \& Molendi (2002) we find that the intrinsic dispersion of the parent population of scaled break radii (assumed to be distributed like a Gaussian) is consistent with 0 (for both SCDM50 and $\Lambda$ CDM70). By performing the same analysis using deprojected temperature profiles we find 0.12 and 0.09 for the mean value of $x_{\mathrm{b}}^{\text {deproj }}$ for SCDM50 and $\Lambda$ CDM70, respectively, and standard deviation of 0.01 . This latter results shows consistency between break radii of projected and deprojected profiles.

\subsection{The outer region}

For comparison with other studies, we quantify the decline seen in Fig. 1 (i.e. in a SCDM50 cosmology) for radii larger 


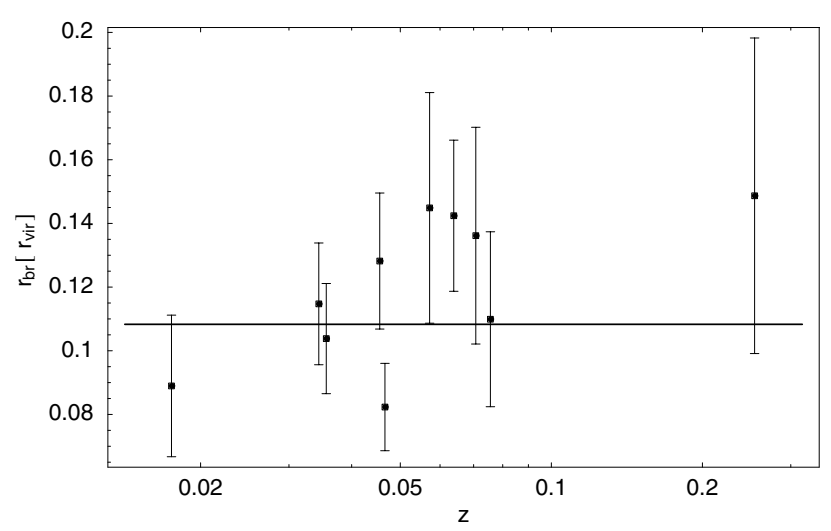

Fig. 3. Break radii $r_{\mathrm{br}}^{\mathrm{proj}}$ in units of $r_{\mathrm{vir}}$ as a function of redshift in SCDM50. The horizontal line is the error-weighted mean value.

than $\sim 0.15 r_{\text {vir }}$ by simply modeling the data with a line. From the data shown in Fig. 1 we select, for each object, bins at distances larger than $r_{\mathrm{br}}^{\text {deproj }}=0.12 r_{\text {vir }}$. We fit the selected points to a straight line and find a slope equal to $-0.97 \pm 0.14$ $\left(\chi^{2}=20.38\right.$, d.o.f. $\left.=34\right)$.

Since many authors use projected temperature profiles, we apply the same procedure to the projected temperature profiles. These are scaled by $r_{180}$ and normalized by the mean emission-weighted temperature $\left\langle T_{\mathrm{X}}^{\mathrm{proj}}\right\rangle$, i.e. computed using the projected data. In this case we obtain $-0.94 \pm 0.11\left(\chi^{2}=26.24\right.$, d.o.f. = 35) for the slope.

For a fair comparison with De Grandi \& Molendi (2002), we compute the value for the slope using their procedure: we use projected temperature profiles (normalized by $\left\langle T_{\mathrm{X}}^{\mathrm{proj}}\right\rangle$ and scaled by $r_{180}^{\mathrm{Ev}}$ ), exclude temperature bins within the cooling region and fit the data with the broken line model given in Eq. (1) in De Grandi \& Molendi (2002), i.e. with a constant $t_{\mathrm{b}}$ up to a break radius $x_{\mathrm{b}}$ and with a line with slope $m$ beyond $x_{\mathrm{b}}$. In excellent agreement with De Grandi \& Molendi (2002), we find $t_{\mathrm{b}}=1.02 \pm 0.02, x_{\mathrm{b}}=0.10 \pm 0.05$ and $\mathrm{a}$ slope $m=-1.15 \pm 0.10$. We also model the profiles scaled in the SCDM50 cosmology with a polytropic equation of state, $T /\left\langle T_{\mathrm{X}}\right\rangle=T_{0}\left(1+\left(r / a_{\mathrm{X}}\right)^{2}\right)^{-3 \beta(\gamma-1) / 2}$. We take values for $\beta$ and $a_{\mathrm{x}}$ from the literature: for Perseus (A 426) from Ettori et al. (1998), for A 3112 from Sanderson et al. (2003) and from Peterson et al. (2003) for the remaining clusters. We then use the mean values $\beta=0.69, a_{\mathrm{x}}=0.087 r_{180}$ in the fit. For $r>r_{\text {br }}^{\text {deproj }}$ we find $T_{0}=1.36 \pm 0.07$ and $\gamma=1.24 \pm 0.03$, in agreement with the results of Markevitch et al. (1998).

Recent numerical simulation are in general able to produce temperature profiles quite similar to the observed ones at large radii (see De Grandi \& Molendi 2002, in particular Fig. 12, for a comparison with simulations results published before 2001). Recently, De Grandi \& Molendi (2002)'s profiles have been compared with simulated profiles of Valdarnini (2003), Borgani et al. (2002) and Borgani et al. (2004), and for radii larger that $\approx 0.15 r_{\text {vir }}$ it was found good agreement. Given the agreement between De Grandi \& Molendi (2002)'s profiles and ours at large cluster-centric distances, these simulations should provide a good description of the temperature profiles presented here as well. Here, we compare the projected

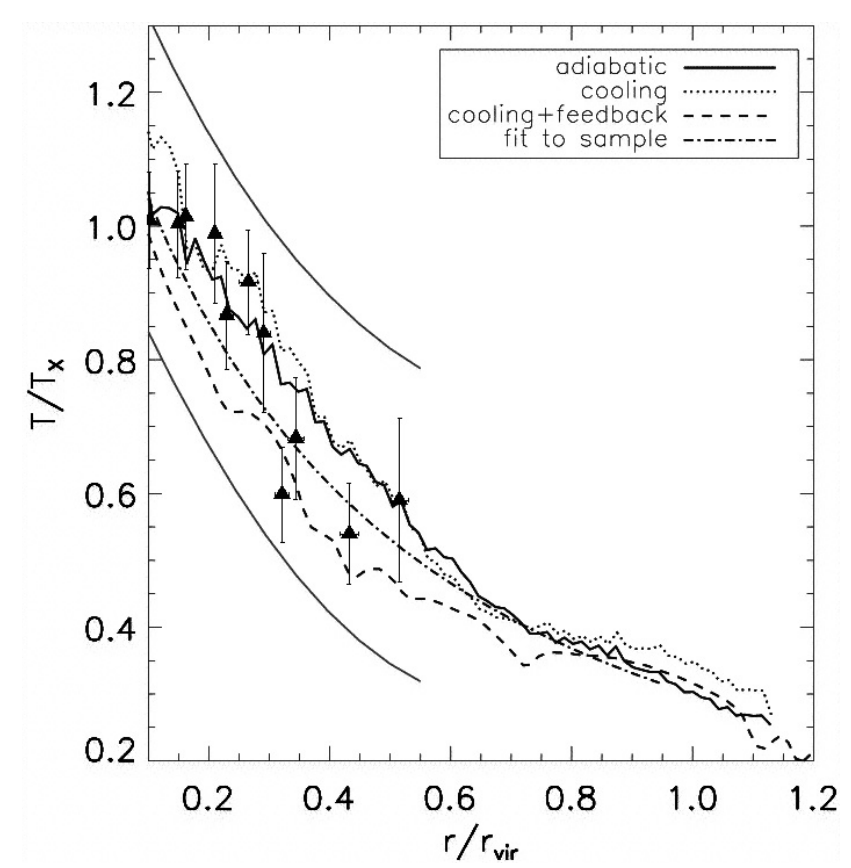

Fig. 4. Mean error-weighted projected temperature profile for our sample (filled triangles, with $1 \sigma$ errors) as a function of radius in units of $r_{\text {vir }}$ together with the results shown in Fig. 6 of Loken et al. (2002). The two solid lines define the approximate band that encloses most temperature profiles and their error bars of Markevitch et al. (1998).

temperature profiles with the numerical predictions by Loken et al. (2002) and find good agreement. Loken et al. (2002) compared their results and found good agreement with observations of Markevitch et al. (1998) and the BeppoSAX profiles presented in De Grandi \& Molendi (2002). The comparison with Loken et al. (2002)'s results is shown in Fig. 4, where we plot the mean error-weighted projected profile for $r>0.1 r_{\mathrm{vir}}$ for our sample of 13 cooling flow clusters on top of the results shown in Fig. 6 of Loken et al. (2002). Loken et al. (2002) find that the simulated clusters in their catalogs (both $\Lambda \mathrm{CDM}$ and SCDM catalogs) show temperature profiles which can be well described by a universal temperature profile of the form $T /\left\langle T_{\mathrm{X}}\right\rangle=T_{0}\left(1+r / a_{\mathrm{x}}\right)^{-\delta}$, with $T_{0}=1.33, a_{\mathrm{x}}=r_{\mathrm{vir}} / 1.5$ and $\delta=1.6$. This profile is clearly not suitable to reproduce the profile typical of cooling flow cluster over the entire observed radial range and a central region must be excised. The radial range used by Loken et al. (2002) in the fit is (0.04-1.00) $r_{\mathrm{vir}}$. Due to the limited radial range of our data, we fit the universal profile found by Loken et al. (2002) to the projected temperature profiles for $\left\langle r_{\mathrm{br}}^{\mathrm{proj}} / r_{180}\right\rangle=0.11 \leq r / r_{180} \leq r_{\max } / r_{180}=0.49$ by assuming $a_{\mathrm{x}}=r_{180} / 1.5$. We find $T_{0}=1.31 \pm 0.05$ and $\delta=1.67 \pm 0.20$. Despite the good agreement, we stress that a more robust comparison will be only possible with observations spanning a much larger radial range than the one used in the present work.

\subsection{The inner region}

The temperature decline towards the center is characteristic of cooling flow clusters. For the sample in this paper, the 
structure of deprojected temperature profiles has been extensively analyzed in Kaastra et al. (2004). The parametrization:

$T(r)=T_{\mathrm{c}}+\left(T_{\mathrm{h}}-T_{\mathrm{c}}\right) \frac{\left(r / r_{\mathrm{c}}\right)^{\mu}}{1+\left(r / r_{\mathrm{c}}\right)^{\mu}}$,

is found to provide a remarkably good description of the temperature decline, with $T_{\mathrm{c}}$ set equal to the temperature of the innermost bin and $\mu=2$. In SCDM50 a correlation between the fitting parameters $T_{\mathrm{h}}$ and $r_{\mathrm{c}}$ of the form $r_{\mathrm{c}}=(4.1 \pm$ $0.8) T_{\mathrm{h}}^{1.84 \pm 0.14}$, with $r_{\mathrm{c}}$ in $\mathrm{kpc}$ and $T_{\mathrm{h}}$ in $\mathrm{keV}$, is found. Assuming that $r_{\Delta}$ scales with $T_{\mathrm{h}}^{1 / 2}$ one concludes that the characteristic radius $r_{\mathrm{c}}$ does not simply scale with the virial radius.

In the following we further explore this implication by considering the mean emission-weighted temperature $\left\langle T_{\mathrm{X}}\right\rangle$ instead is $T_{\mathrm{h}}$ and using the estimated $r_{\Delta}$ instead of assuming $r_{\Delta} \propto T_{\mathrm{h}}^{1 / 2}$. For both SCDM50 and $\Lambda$ CDM70 cosmologies we find that the characteristic length scale $r_{\mathrm{c}}$ is not linked to the size of the dark matted halo hosting the cooling flow in a simple way. In SCDM50, for example, we find $r_{\mathrm{c}} \propto\left\langle T_{\mathrm{X}}\right\rangle^{1.78 \pm 0.10}$ and $r_{2500} \propto$ $\left\langle T_{\mathrm{X}}\right\rangle^{0.88 \pm 0.02}$, with $r_{\mathrm{c}}$ and $r_{2500}$ in $\mathrm{kpc}$ and $\left\langle T_{\mathrm{X}}\right\rangle$ in $\mathrm{keV}$. In this context we notice that the flattening of the composite scaled temperature profile in the range $\sim 0.1-0.2 r_{\mathrm{vir}}$ in SCDM50 (see Fig. 1) is artificial, as it is due to the fact that temperature profiles in the inner region do not simply scale with the virial radius.

Kaastra et al. (2004) searched for correlations between parameters of the cooling gas without finding any significant one (Kaastra et al. 2004, Sect. 4.1). Having additional information about the characteristic radii $r_{\Delta}$, we investigated correlations of these with the parameters of the cooling gas, such as relative temperature decrement and cooling radius. We do not find any significant correlation.

As shown in the previous section, for radii larger than $\sim 0.15 r_{\mathrm{vir}}$ most of the numerical simulations are able to produce temperature profiles quite similar to the ones presented in this work. The fair comparison with simulated cluster temperature profiles in the central region, i.e. for radii smaller than the break radius $r_{\mathrm{br}}$, is not possible yet. In fact, the central temperature drop has not been reproduced consistently by any simulation (e.g., Tornatore et al. 2003; Borgani et al. 2004, and references therein). In this context, our temperature parametrization for the inner region of cooling flow clusters (Eq. (4)) and the correlation between its free parameters $\left(r_{\mathrm{c}} \propto\left\langle T_{\mathrm{X}}\right\rangle^{1.78 \pm 0.10}\right)$ provides an important test for forthcoming simulation models.

\section{Entropy profiles and the $L-T$ relation}

The entropy is a useful quantity to probe the thermodynamic history of the hot gas in groups and clusters. As it has become customary, we define the entropy as $S=k T / n_{\mathrm{e}}^{2 / 3}$, where $T$ and $n_{\mathrm{e}}$ are the deprojected electron temperature and density, respectively. $S$, which is by some authors referred as to "entropy index", is related to the thermodynamic entropy via a logarithm and an additive constant and therefore conserved in any adiabatic process.

Particular interest has been given to the shape of entropy profiles in groups, since preheating models predict both large isentropic cores and a break in the entropy temperature relation

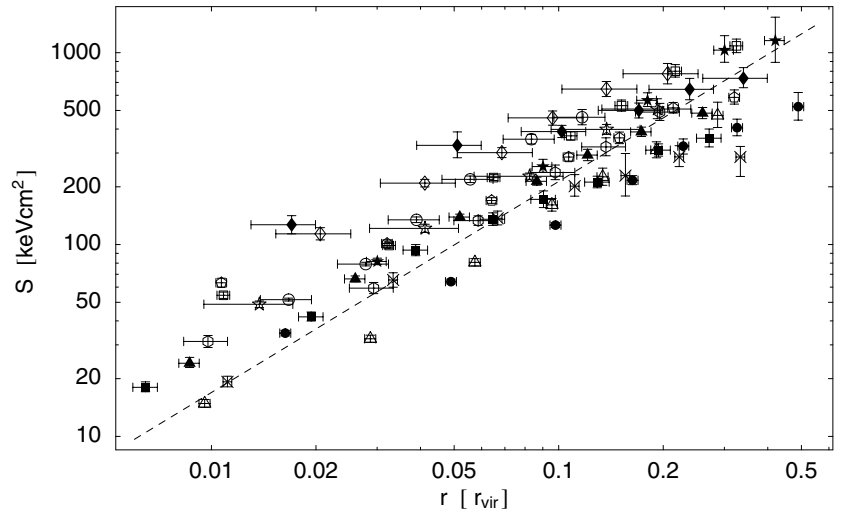

Fig. 5. Entropy profiles in SCDM50: the radius is scaled with the radius $r_{\text {vir }}=r_{180}$, while no scaling has been applied to the entropy values. Clusters are related to symbols as follows: NGC 533 (crosses), A 262 (filled squares), A 1837 (filled diamonds), Sérsic 159-3 (filled circles), 2A 0335+096 (open triangles), MKW 3s (open pentagons), A 2052 (filled triangles), A 4059 (open diamonds), A 496 (open hexagon), A 3112 (open stars), A 1795 (open squares), Perseus (open circles) and A 1835 (filled stars). The dashed line indicates the best fit power-law with the power index value fixed to 1.1 predicted by Tozzi \& Norman (2001).

(e.g., Tozzi \& Norman 2001). While there is evidence that even at group scale no large isentropic cores are present (Pratt \& Arnaud 2003; Ponman et al. 2003), the entropy profiles of cooling flow clusters are expected to increase monotonically moving outwards and to show no isentropic cores (e.g., McCarthy et al. 2004).

In Fig. 5 we plot entropy profiles for the 13 cooling flow clusters with the radial coordinate scaled by $r_{\mathrm{vir}}$ in the SCDM50 cosmology. A visual inspection of the individual profiles in the plot shows that, as expected for systems with short cooling times in their central regions, no cores are present and the entropy of the ICM increases monotonically moving outwards.

In the standard self-similar scenario, one predicts $S \propto$ $h^{-4 / 3}(z) T$, where $h^{2}(z)=\Omega_{\mathrm{m}}(1+z)^{3}+\Omega_{\Lambda}$ (e.g., Pratt \& Arnaud 2005). On the other hand, recent results by Ponman et al. (2003) suggest that entropy scales with temperature as $S \propto T^{0.65}$, the so-called "entropy ramp", instead of $S \propto T$. Both scalings $S \propto h^{-4 / 3}(z) T$ and $S \propto h^{-4 / 3}(z) T^{0.65}$ considerably reduce the dispersion of the profiles shown in Fig. 5. Using $r_{\mathrm{vir}}$ to scale radii, we quantify the dispersion of the scaled profiles using the standard deviation and the mean of the scaled entropy values at a fixed scaled radius. As mean cluster temperature $T$, the mean emission-weighted temperature $\left\langle T_{\mathrm{X}}\right\rangle$ (see Table 2) is used. The scaled entropy values are evaluated at fractions of $r_{\mathrm{vir}}$ for which no extrapolation is needed and we compute the ratio between their standard deviation $\sigma$ and mean $m$ to quantify the relative dispersion of the scaled profiles. For both SCDM50 and $\Lambda$ CDM70 cosmologies we find that the dispersion is less if the "entropy ramp" scaling is used. For SCDM50 we find $\sigma / m=0.37$ and $\sigma / m=0.29$ at $0.1 r_{\text {vir }}$ for the $S \propto T$ and $S \propto T^{0.65}$ temperature scaling, respectively. The relative difference of the $\sigma / m$ ratios is the same in $\Lambda$ CDM70 and the relative dispersion $\sigma / m$ varies with radius only by a few percent (e.g. 


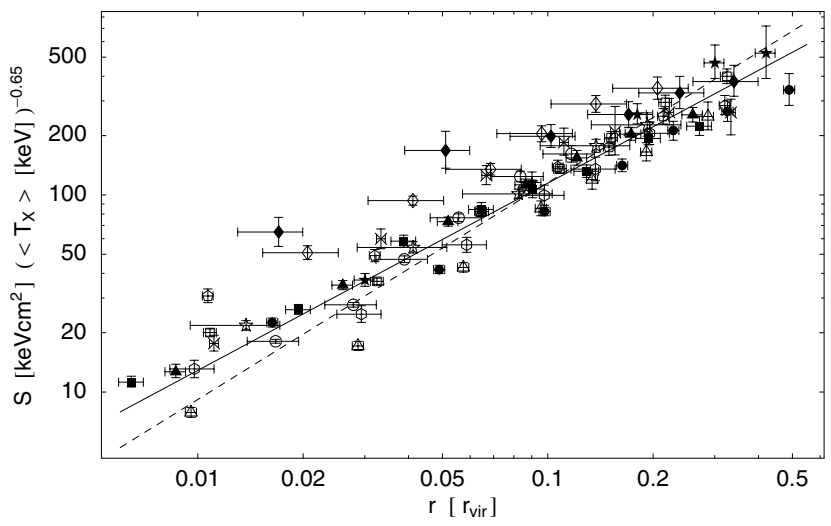

Fig. 6. Same as Fig. 5 but with the scaling $S \propto h^{-4 / 3}(z) T^{0.65}$ used instead. The solid line indicates the best fit power-law (best fit power index 0.95) and the dashed one the best fit power-law with the power index value fixed to 1.1 .

$5 \%$ between 0.03 and $0.2 r_{\text {vir }}$ in SCDM50). From scalings of the entropy profiles of 5 clusters (A 1983, A 2717, MKW9, A 1991 and A 1413), Pratt \& Arnaud (2005) also find that the scatter in the scaled entropy profiles is smaller if one uses Ponman et al. (2003)'s scaling rather than the standard self-similar one. In addition to the dispersion analysis, we attempted to gain further information on the scaling of entropy with temperature by directly fitting the $S-T$ data (i.e. $S\left(0.1 r_{200}\right)$ versus $\left.\left\langle T_{\mathrm{X}}\right\rangle\right)$, but due to the small sample size and the fact that the systems do not span a wide temperature range, we do not obtain any statistically significant constrain on its slope. The profiles scaled using the relation $S \propto h^{-4 / 3}(z) T^{0.65}$ are shown in Fig. 6 for SCDM50. We fit the data shown in Fig. 6 with a line in $\log -\log$ space (with errors in both coordinates) and find, in excellent agreement with the value found by Pratt \& Arnaud (2005), a slope equal to $0.95 \pm 0.02$. Similarly, using Chandra data, Ettori et al. (2002a) find a slope equal to 0.97 for the entropy profile of A 1795. Therefore, our analysis gives additional evidence for a slope close to, but slightly shallower than 1.1 , the value predicted by shock dominated spherical collapse models (Tozzi \& Norman 2001). This behavior is also a common feature of numerical simulations (see Pratt \& Arnaud 2005, and references therein).

From the scaled entropy profiles we can obtain an estimate of the normalization of the $S \propto T^{0.65}$ relation found by Ponman et al. (2003). For the entropy profiles with entropy values scaled as $S \propto h^{-4 / 3}(z)(T / 10 \mathrm{keV})^{0.65}$ and the radial coordinate scaled by $r_{200}$ we find a mean value at $0.1 r_{200}$ equal to 504 (with standard deviation 140). The latter value is in excellent agreement with the normalization of Ponman et al. (2003)'s $S\left(0.1 r_{200}\right)-T$ relation at $T=10 \mathrm{keV}$ (see their Fig. 5).

Although the relation $S \propto T^{0.65}$ provides evidence for similarity-breaking, the most robust evidence for the lack of self-similarity in the ICM properties is provided by the luminosity-temperature $(L-T)$ relation. The $L \propto T^{\alpha}$ law, with $\alpha=2$ expected for self-similar systems is at variance with observations at both cluster and group scales. For clusters, most authors (e.g. White et al. 1997; Arnaud \& Evrard 1999) have found $\alpha \sim 3$, while for groups there is evidence for a much steeper slope $\alpha \sim 5$ (e.g. Helsdon \& Ponman 2000a,b;

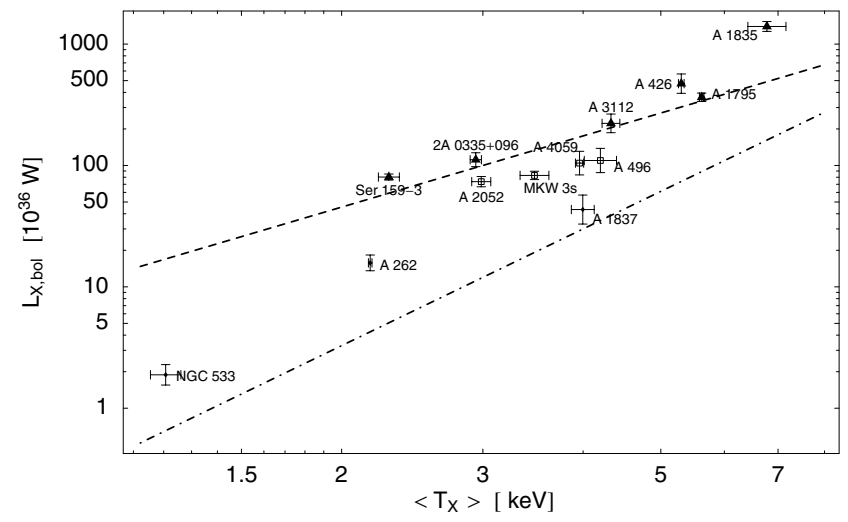

Fig. 7. The cooling flow "uncorrected" $L-T$ relation in SCDM50. The plotted quantities are the mean emission-weighted temperature $\left\langle T_{\mathrm{X}}\right\rangle$ and the bolometric X-ray luminosity within $r_{500}$. Filled triangles and open boxes indicate clusters with $\dot{M}>300 M_{\odot} \mathrm{yr}^{-1}$ and $\dot{M}>$ $100 M_{\odot} \mathrm{yr}^{-1}$, respectively. Mass deposition rates for A 1837 are not available in the literature, while $\dot{M}$ for A 262 and NGC 533 are consistent with zero. The dashed line shows McCarthy et al. (2004) predictions for an entropy injection level of $200 \mathrm{keV} \mathrm{cm}$, while the dot-dashed line an entropy injection level of $500 \mathrm{keV} \mathrm{\textrm {cm } ^ { 2 }}$.

Xue \& Wu 2000). The latter result is however not confirmed by the work of Mulchaey \& Zabludoff (1998) and most important by the recent analysis by Osmond \& Ponman (2004), as both works indicate that the $L-T$ relation at group scales is consistent with and extrapolation of the trend observed at cluster scale $(\alpha \sim 3)$.

A great deal of semi-analytical and numerical studies have attempted to give an explanation to the observed similarity-breaking. While it is clear that models incorporating gravitationally-driven processes only fail to match observations, there is evidence that models incorporating a mixture of both cooling and heating processes are likely to be successful (see Ponman et al. 2003, and references therein). Such models should of course also explain the properties of cooling flow clusters. Cooling flow clusters are known to enhance the scattering of the observed $L-T$ relation, which is reduced once the contribution from the cooling region is excised or cooling flow clusters are excluded from the sample (Markevitch 1998; Ettori et al. 2002b). On the other hand, the scatter in not random and theoretical models incorporating gas cooling should be compared to "uncorrected" data. This kind of comparison has never been presented in semi-analytical or numerical works until recently in McCarthy et al. (2004)'s work. McCarthy et al. (2004) have extended the work by Babul et al. (2002) to include radiative cooling and compared their predictions with observations without any "cooling flow" correction.

In Fig. 7 we show the $L-T$ relation in SCDM50 for the 13 cooling flow clusters we have analyzed. According to McCarthy et al. (2004)'s predictions, clusters with large mass deposition rates $\dot{M}$ lie on the high luminosity side of the scaling relation. We take mass deposition rates from Peterson et al. (2003) and, for the objects not present in the latter work (A 3112 and A 426) from Peres et al. (1998). Mass deposition rates for A 1837 are not available in the literature. We divide the clusters into three groups: those with $\dot{M}>300 M_{\odot} \mathrm{yr}^{-1}$ 


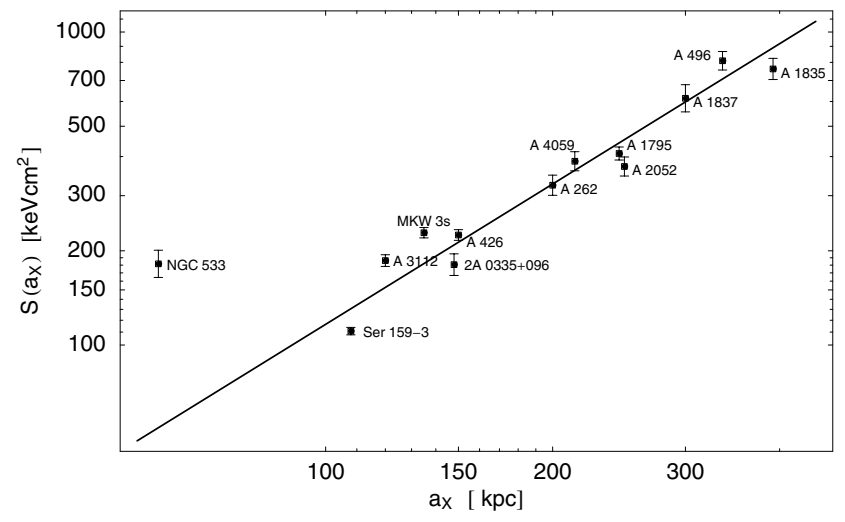

Fig. 8. The core radius-central entropy relation in SCDM50. The solid line indicates the best fit power law. NGC 533 is excluded from the fit.

("massive cooling flow clusters"), with $\dot{M}>100 M_{\odot} \mathrm{yr}^{-1}$ ("moderate cooling flow clusters") and clusters with mass deposition rates consistent with zero. Even though our sample is small, a clear trend consistent with the findings of McCarthy et al. (2004) can be seen from a visual inspection of Fig. 7, in which we also show the predictions by McCarthy et al. (2004) for an entropy injection level of $200 \mathrm{keV} \mathrm{cm}$ and $500 \mathrm{keV} \mathrm{cm}$ (models evolved for 10 Gyrs, see Babul et al. 2003). "Massive cooling flow clusters" are consistent with being systems that experienced "mild" ( $\left.200 \mathrm{keV} \mathrm{cm}{ }^{2}\right)$ levels of entropy injection.

We feel it should be pointed out that the entropy injection levels in McCarthy et al. (2004)'s model are quite high compared to most of the preheating models (e.g., see Voit et al. 2003, and references therein). However, a comparison between preheating models is beyond the scope of our work, being our main purpose that of providing "uncorrected" $L-T$ data for a sample of cooling flow clusters. In Fig. 8 we show a second relationship predicted by the work of McCarthy et al. (2004): the correlation between core radius and central entropy. As expected, we find that the entropy at the core radius $a_{\mathrm{X}}$ increases with increasing core radius, with the exception of the low mass system NGC 533. Using core radii taken from the literature (see above) and excluding NGC 533 from the fit, we find: $\log \left[S\left(a_{\mathrm{X}}\right)\right]=1.48 \log \left[a_{\mathrm{X}}\right]-0.91$ (entropy in $\mathrm{keV} \mathrm{cm}{ }^{2}$ and core radius in $\mathrm{kpc})$.

\section{Summary and conclusions}

We have investigated temperature and entropy profiles of cooling flow clusters using the results from the spatially resolved spectra taken with the EPIC cameras of XMM-Newton presented in Kaastra et al. (2004).

Using deprojected plasma densities and temperatures we compute the total gravitational mass profile and the virial radius of each clusters and find that:

- The inclusion of the mass contribution from the gas in the estimate of the total mass of the cluster is very important and gives more robust estimates.
- As recently found by other authors, the estimated virial radii substantially deviate from those estimated assuming the standard size-temperature relation.

- Our total and gas mass estimates are in good agreement with published ASCA and BeppoSax estimates.

Our main findings concerning the temperature profiles are:

- When normalized and scaled by the virial radius, the temperature profiles are, except in the central cooling region, remarkably similar.

- The profiles show a break radius $r_{\mathrm{br}} \sim 0.12$ and $0.09 r_{\mathrm{vir}}$ in SCDM50 and $\Lambda$ CDM70 cosmology, respectively.

- Most important, the temperature profiles show a decline beyond the break radius.

- Our findings for the shape and slope of the profiles in this range is in excellent agreement with previous studies by Markevitch et al. (1998) and De Grandi \& Molendi (2002). In SCDM50, a linear fit to the deprojected temperature profiles with cluster-centric distance scaled by the virial radius, gives a slope of $-0.97 \pm 0.14$ between $\sim 0.15 r_{\mathrm{vir}}$ and $0.50 r_{\text {vir. }}$.

- For radii larger than $\sim 0.15 r_{\text {vir }}$ (in SCDM50) most of the numerical simulations are able to produce temperature profiles quite similar to the observed ones.

- The temperature decline from $r_{\mathrm{br}}$ inwards is very well parametrized using a profile with a core $r_{\mathrm{c}}$ (see Eq. (4)). We find that $r_{\mathrm{c}} \propto\left\langle T_{\mathrm{X}}\right\rangle^{1.78 \pm 0.10}$, with the mean cluster temperature $\left\langle T_{\mathrm{X}}\right\rangle$ in $\mathrm{keV}$ and $r_{\mathrm{c}}$ in $\mathrm{kpc}$, and that $r_{\mathrm{c}}$ is not proportional to the cluster virial radius.

We have studied entropy profiles and find that:

- As expected for cooling flow clusters, i.e. massive systems with strong cooling, no isentropic cores are present.

- The entropy of the gas increases monotonically moving outwards almost proportional to the radius.

- The scatter in the scaled entropy profiles is smaller if the empirical relation $S \propto T^{0.65}$ (Ponman et al. 2003) is used instead of the standard self-similar relation $S \propto T$.

- The profiles are close to, but slightly shallower $\left(\propto r^{0.95}\right.$ with entropies scaled using $S \propto T^{0.65}$ ) than those predicted by analytical models of shock dominated spherical collapse $\left(\propto r^{1.1}\right)$.

- The normalization of the $S-T$ relation derived from the scaling of the entropy profiles is in excellent agreement with the one found by Ponman et al. (2003).

- We confirm the existence of a tight correlation between core radius and central entropy.

Acknowledgements. We wish to thank the referee for insightful comments which improved the presentation of the results. This work is based on observations obtained with XMM-Newton, an ESA science mission with instruments and contributions directly funded by ESA Member States and the USA (NASA). R.P. acknowledges partial support by the Swiss National Science Foundation.

\section{References}

Allen, S. W., Schmidt, R. W., \& Fabian, A. C. 2001, MNRAS, 328, L37 
Arnaud, M., \& Evrard, A. E. 1999, MNRAS, 305, 631

Arnaud, M., Neumann, D. M., Aghanim, N., et al. 2001, A\&A, 365, L80

Arnaud, M., Majerowicz, S., Lumb, D., et al. 2002, A\&A, 390, 27

Babul, A., Balogh, M. L., Lewis, G. F., \& Poole, G. B. 2002, MNRAS, 330,329

Babul, A., McCarthy, I. G., \& Poole, G. B. 2003, to appear in the proceedings of the Multiwavelength Cosmology, Conf. held in Mykonos, Greece, June 2003, ed. M. Plionis (Kluwer) [arXiv: astro-ph/0309543]

Borgani, S., Governato, F., Wadsley, et al. 2002, MNRAS, 336, 409 Borgani, S., Murante, G., Springel, V., et al. 2004, MNRAS, 348, 1078 Bryan, G. L., \& Norman, M. L. 1998, ApJ, 495, 80

De Grandi, S., \& Molendi, S. 2002, ApJ, 567, 163

Ettori, S., Fabian, A. C., \& White, D. A. 1998, MNRAS, 300, 837

Ettori, S., Fabian, A. C., Allen, S. W., \& Johnstone, R. M. 2002a, MNRAS, 331, 635

Ettori, S., De Grandi, S., \& Molendi, S. 2002b, A\&A, 391, 841

Evrard, A. E., Metzler, C. A., \& Navarro, J. F. 1996, ApJ, 469, 494

Finoguenov, A., Reiprich, T. H., \& Böhringer, H. 2001a, A\&A, 368, 749

Finoguenov, A., Arnaud, M., \& David, L. P. 2001b, ApJ, 555, 191

Helsdon, S. F., \& Ponman, T. J. 2000a, MNRAS, 315, 356

Helsdon, S. F., \& Ponman, T. J. 2000b, MNRAS, 319, 933

Irwin, J. A., Bregman, J. N., \& Evrard, A. E. 1999, ApJ, 519, 518

Irwin, J. A., \& Bregman, J. N. 2000, ApJ, 538, 543

Kaastra, J. S., Tamura, T., Peterson, J. R., et al. 2004, A\&A, 413, 415

Loken, C., Norman, M. L., Nelson, E., et al. 2002, ApJ, 579, 571

Majerowicz, S., Neumann, D. M., \& Reiprich, T. H. 2002, A\&A, 394, 77

Markevitch, M. 1998, ApJ, 504, 27

Markevitch, M., Forman, W. R., Sarazin, C. L., \& Vikhlinin, A. 1998, ApJ, 503, 77

McCarthy, I. G., Balogh, M. L., Babul, A., et al. 2004, ApJ, accepted [arXiv: astro-ph/0406329]

Mohr, J. J., \& Evrard, A. E. 1997, ApJ, 491, 38
Mulchaey, J. S., \& Zabludoff, A. I. 1998, ApJ, 496, 73

Navarro, J. F., Frenk, C. S., \& White, S. D. M. 1996, ApJ, 462, 563

Osmond, J. P. F., \& Ponman, T. J. 2004, MNRAS, 350, 1511

Peres, C. B., Fabian, A. C., Edge, A. C., et al. 1998, MNRAS, 298, 416

Peterson, J. R., Kahn, S. M., Paerels, F. B. S., et al. 2003, ApJ, 590, 207

Ponman, T. J., Sanderson, A. J. R., \& Finoguenov, A. 2003, MNRAS, 343,331

Pratt, G. W., Arnaud, M., \& Aghanim, N. 2001, in XXIst Moriond Astrophysics Meeting, Clusters of Galaxies and the High Redshift Universe Observed in X-rays, ed. D. M. Neumann, J. Tranh Thanh Van, published electronically at http://www-dapnia.cea.fr/Conferences/ Morion_astro_2001/[arXiv:astro-ph/0105431]

Pratt, G. W., \& Arnaud, M. 2002, A\&A, 394, 375

Pratt, G. W., \& Arnaud, M. 2003, A\&A, 408, 1

Pratt, G. W., \& Arnaud, M. 2005, A\&A, 429, 791

Rephaeli, Y. 1995, ARA\&A, 33, 541

Sanderson, A. J. R., Ponman, T. J., Finoguenov, A., Lloyd-Davies, E. J., \& Markevitch, M. 2003, MNRAS, 340, 989

Takahashi, S., \& Yamashita, K. 2003, PASJ, 55, 1105

Tornatore, L., Borgani, S., Springel, V., et al. 2003, MNRAS, 342, 1025

Tozzi, P., \& Norman, C. 2001, ApJ, 546, 63

Valdarnini, R. 2003, MNRAS, 339, 1117

Voit, G. M., Balogh, M. L., Bower, R. G., Lacey, C. G., \& Bryan, G. L. 2003, ApJ, 593, 272

Voit, M. G. 2004, Rev. Mod. Phys., in press [arXiv:astro-ph/0410173]

White, S. D. M., Navarro, J. F., Evrard, A. E., \& Frenk, C. S. 1993, Nature, 366, 429

White, D. A., Jones, C., \& Forman, W. 1997, MNRAS, 292, 419

White, D. A. 2000, MNRAS, 312, 663

Xue, Y., \& Wu, X. 2000, ApJ, 538, 65 